\title{
Increased creatine kinase MB level predicts postoperative mortality after cardiac surgery independent of new 0 waves
}

\author{
James Ramsay, $\mathrm{MD}^{\mathrm{a}}$ \\ Stanton Shernan, $M D^{b}$ \\ Jane Fitch, MD ${ }^{c}$ \\ Paul Finnegan, $M D^{d}$ \\ Thomas Todaro, MD, JDe \\ Thomas Filloon, $\mathrm{PhD}^{\mathrm{e}}$ \\ Nancy A. Nussmeier, $M D^{f}$
}

A supplementary appendix is available online. $\beta$
Background: Recent consensus statements recommend cardiac enzyme release as the essential criterion for diagnosing myocardial infarction. However, the outcome implications of cardiac enzyme release in patients undergoing coronary artery bypass grafting are controversial.

Methods: Eight hundred patients were followed for 30 days after elective on-pump coronary artery bypass grafting in a multicenter, prospective, randomized trial of the anti-C5 complement antibody pexelizumab. Data from centralized electrocardiography and creatine kinase MB analyses were examined for any association with death or severe left ventricular dysfunction.

Results: More than half of the 800 patients had peak creatine kinase MB levels of more than 5 times the upper limit of $5 \mathrm{ng} / \mathrm{mL}$ set by the core laboratory. The median peak value was $29 \mathrm{ng} / \mathrm{mL}$. The incidence of the combined outcome (death or severe left ventricular dysfunction) was $1.7 \%$ if the peak creatine kinase MB level was less than $25 \mathrm{ng} / \mathrm{mL}$ and $18.0 \%$ if $100 \mathrm{ng} / \mathrm{mL}$ or greater $(P<.01)$. Similarly, the incidence of new Q-wave myocardial infarction was 3.9\% if the peak creatine kinase MB level was less than $25 \mathrm{ng} / \mathrm{mL}$ and $30.6 \%$ if $100 \mathrm{ng} / \mathrm{mL}$ or greater $(P<.01)$. In a multivariate analysis that included preoperative and intraoperative factors, as well as peak enzyme release and Q-wave myocardial infarction, the strongest predictor of the combined outcome was a peak creatine kinase MB level of $100 \mathrm{ng} / \mathrm{mL}$ or greater. New Q-wave myocardial infarction did not significantly predict the combined outcome.

Conclusions: Increased postoperative peak creatine kinase MB level, especially when 20 times or more of the upper limit of normal, indicates increased risk of severe postoperative left ventricular dysfunction and mortality within 30 days of coronary artery bypass grafting. High peak enzyme level is a stronger predictor of adverse outcomes than is postoperative Q-wave myocardial infarction in this population.

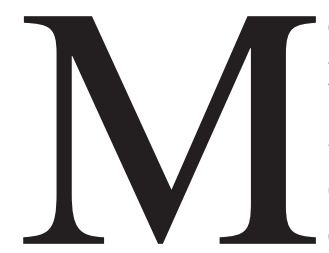

ethods of diagnosing myocardial infarctions (MIs) and their prognostic significance after coronary artery bypass surgery (CABG) have been controversial for 3 decades. Depending on the diagnostic criteria, the reported incidence of MI during CABG ranges from $4 \%$ to $80 \% .{ }^{1}$ New $Q$ waves on the electrocardiogram (ECG), once considered the best available predictor of outcome after $\mathrm{CABG}$, have been variously reported as significant ${ }^{2,3}$ or 
not significant ${ }^{4,5}$ in forecasting long-term survival. This controversy exists partly because ECG changes occur in the majority of patients undergoing $\mathrm{CABG}$, making the significance of these changes difficult to interpret. Myocardial enzyme release, another potential marker of MI, is similarly common in patients undergoing CABG. However, study of the outcome implications of enzyme release in the CABG population has only recently begun, and there is substantial room for refinement.

Two consensus statements by cardiology groups have recommended the use of biochemical markers as the essential criterion for diagnosis of acute MI. ${ }^{6,7}$ Both statements include MI after CABG in this recommendation but cite little supporting evidence and state the need for more data. Since the publication of the consensus statements, reports from 2 prospective perioperative studies addressing this issue have appeared, one of 496 patients randomized to CABG in a study of angioplasty versus CABG surgery ${ }^{8}$ and another of 2394 patients randomized to CABG in a study of the $\mathrm{Na}^{+} / \mathrm{H}^{+}$exchange inhibitor cariporide. ${ }^{9}$ Both reports note statistically significant increases in the risk of death after discharge when peak myocardium-specific creatine kinase (CK) MB isoenzyme levels are greater than 5 times the upper limit of normal. Limitations of these reports include the absence of ECG data from the first ${ }^{8}$ and the absence of central laboratory enzyme analysis from the second. ${ }^{9}$ Here we report data collected from 800 patients undergoing $\mathrm{CABG}$ in a study of the humanized monoclonal antibody pexelizumab, which blocks activation of C5 complement. The results of centralized ECG analysis and central laboratory enzyme analysis are presented.

\section{Methods}

\section{Patients}

The study sample consisted of patients who underwent CABG with cardiopulmonary bypass (CPB) in 1999 and 2000 at one of 65 centers in the United States. These patients were enrolled in a prospective, multicenter, randomized, double-blind, placebo-controlled phase II trial of pexelizumab, a novel single-chain fragment of a humanized monoclonal antibody against complement component C5. ${ }^{10}$ This study was approved by the institutional review board of each participating institution, and informed consent was obtained from all patients.

Patients were included in the study if they had any of the following risk factors for myocardial necrosis: age of 60 years or greater, previous $\mathrm{CABG}$, history of congestive heart failure (New York Heart Association class III or IV), history of more than 2 MIs, an MI within 14 days of screening, history of cerebrovascular disease (cerebrovascular accident, transient ischemic attack, and/or carotid endarterectomy), or a current serum creatinine level of 1.3 $\mathrm{mg} / \mathrm{dL}$ or greater but less than $2.0 \mathrm{mg} / \mathrm{dL}$. Exclusion criteria included active infection or malignancy, uncontrolled diabetes, hepatic disease, preoperative intra-aortic balloon pump or left ventricular (LV) assist device, cardiogenic shock, recent (within 72 hours) percutaneous angioplasty failure, presence of a ventric-
TABLE 1. Demographic and perioperative data for all patients $(\mathbf{n}=\mathbf{8 0 0})$

\begin{tabular}{lc}
\hline Age, y (mean \pm SD) & $67 \pm 9$ \\
Weight, kg (mean \pm SD) & $85 \pm 15$ \\
Sex & \\
$\quad$ Male & $612(76.5)$ \\
$\quad$ Female & $188(23.5)$ \\
Diabetes (type 1 or 2) & $274(34.2)$ \\
Congestive heart failure & $132(16.5)$ \\
Peripheral vascular disease & $147(18.4)$ \\
Hypertension & $627(78.4)$ \\
Tobacco use & $291(36.4)$ \\
Chronic obstructive & $12(1.5)$ \\
pulmonary disease & \\
Previous MI & $393(49.1)$ \\
Renal insufficiency & $147(18.4)$ \\
Saphenous vein graft & $718(89.8)$ \\
Internal mammary artery & $745(93.1)$ \\
graft & \\
Complete revascularization & $762(95.2)$ \\
CPB time, min (mean \pm & $94 \pm 36$ \\
SD) & \\
Aortic crossclamp time, min & $64 \pm 26$ \\
(mean \pm SD) & \\
History of neurologic event & $121(15.1)$ \\
Previous CABG & $63(7.9)$ \\
\hline
\end{tabular}

Data are presented as the number of patients (\%) unless otherwise specified. $M I$, Myocardial infarction; $C P B$, cardiopulmonary bypass; $C A B G$, coronary artery bypass grafting.

ular pacemaker, or complete left bundle branch block on a 12-lead ECG.

Nine hundred fourteen patients were enrolled in the primary study. For this substudy of cardiac enzyme release, patients who underwent combined CABG and valve surgery $(\mathrm{n}=114)$ were excluded, leaving 800 patients undergoing isolated CABG in the present study. Patients were randomized to 1 of 3 treatments: (1) placebo, (2) a $2.0 \mathrm{mg} / \mathrm{kg}$ bolus of pexelizumab after induction of general anesthesia, or (3) a $2.0 \mathrm{mg} / \mathrm{kg}$ bolus of pexelizumab followed by a continuous infusion of $0.05 \mathrm{mg} \cdot \mathrm{kg}^{-1} \cdot \mathrm{h}^{-1}$ for 24 hours. Although the conduct of the anesthesia and surgical intervention was similar for each institution, no attempts were made to standardize techniques. Aortic crossclamping with cardioplegic arrest and CPB using hemodilution, mild-to-moderate hypothermia, and membrane oxygenators were used at all institutions.

\section{Data Collection}

CK-MB levels were measured at a centralized core laboratory facility (Q-LABS, Atlanta, Ga) by using standard techniques. The upper limit of the normal range for this analysis is $5 \mathrm{ng} / \mathrm{mL}$. Blood samples were acquired preoperatively (baseline) and postoperatively at 4, 8, 16, 20,24, 30, and 36 hours after release of the aortic crossclamp and on postoperative days 2, 4, 7, and 30. All bioanalytic and CK-MB assays were validated according to International Conference on Harmonisation guidelines. Although pexelizumab is nonreactive with any other non-C5-complement human tissue or other species complement, the Abbott Axsym CK-MB assay that 


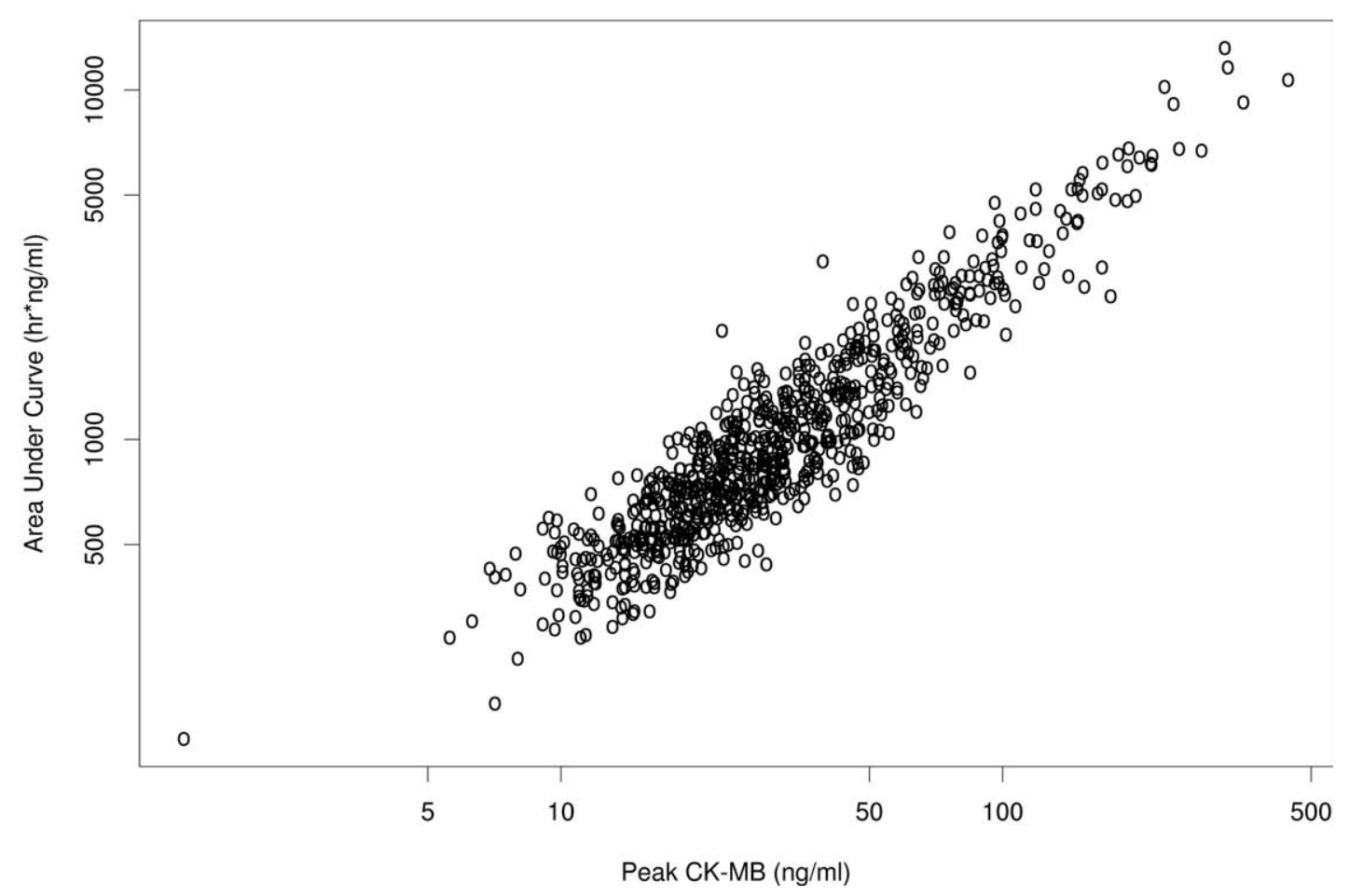

Figure 1. Relationship between AUC for postoperative CK-MB release (hours * nanograms per milliliter) and peak postoperative CK-MB level (nanograms per milliliter).

was used has, since 1998, included blocking antibodies in the reagent and washing steps to prevent any possible interference.

ECGs were obtained preoperatively, on intensive care unit admission, and on postoperative days 1, 4, 7, and 30. A blinded and independent core laboratory (Ischemia Research and Education Foundation, San Francisco, Calif) analyzed ECG records to determine postoperative Q-wave MI according to Minnesota code. $^{11}$

\section{Statistical Analysis}

Analyses were performed to examine the relationship between peak CK-MB release and 30-day mortality or severe LV dysfunction (defined as use of $\geq 4$ inotropic drugs, LV assist device, or intra-aortic balloon pump). The area under the curve (AUC) for CK-MB release was determined with the linear trapezoidal method by using each patient's available data (with linear interpolation for missing intermediate time points). The relationship between AUC and peak CK-MB level was explored with Spearman rank correlation. Patients were then grouped by CK-MB level, and contingency table analysis and $\chi^{2}$ tests were used to compare event rates across these groups. The Cochran-Mantel-Haenszel trend test was used to check for any association between peak levels of CK-MB and new $\mathrm{Q}$ waves on the postoperative ECG.

Univariate and multiple logistic regression were used to construct a model of the association between perioperative variables and the risk of 30-day mortality or severe LV dysfunction, as defined above. Univariate logistic regression analysis was per- formed on each of several candidate variables. If the subsequent $P$ value was less than .15 , then the variable was included in an initial multiple logistic regression model. Variables were omitted from the final multiple regression model if their $P$ value in the initial model was greater than .10 .

\section{Results}

\section{Demographic and Perioperative Data}

Preoperative patient characteristics and intraoperative data (Table 1) were similar across the 3 study groups, and there were no differences between groups in the rate of the primary composite endpoint of death, MI, ventricular dysfunction, and central neurologic deficit. ${ }^{10} \mathrm{~A}$ post hoc analysis demonstrated that patients who received a bolus injection plus subsequent infusion of the C5 complement inhibitor had a lower risk of the composite endpoint of CK-MB of $100 \mathrm{ng} / \mathrm{mL}$ or greater or death within 30 days of CABG than did placebo or bolus-only patients. ${ }^{10}$

\section{CK-MB Release}

For the remaining analyses, patients in all 3 study groups were combined into a single 800-patient cohort. AUC and peak CK-MB values in this cohort showed a very strong linear relationship (Spearman $r=0.9 ; P<.005$; Figure 1). Consequently, the peak CK-MB value was used in all subsequent analyses. 


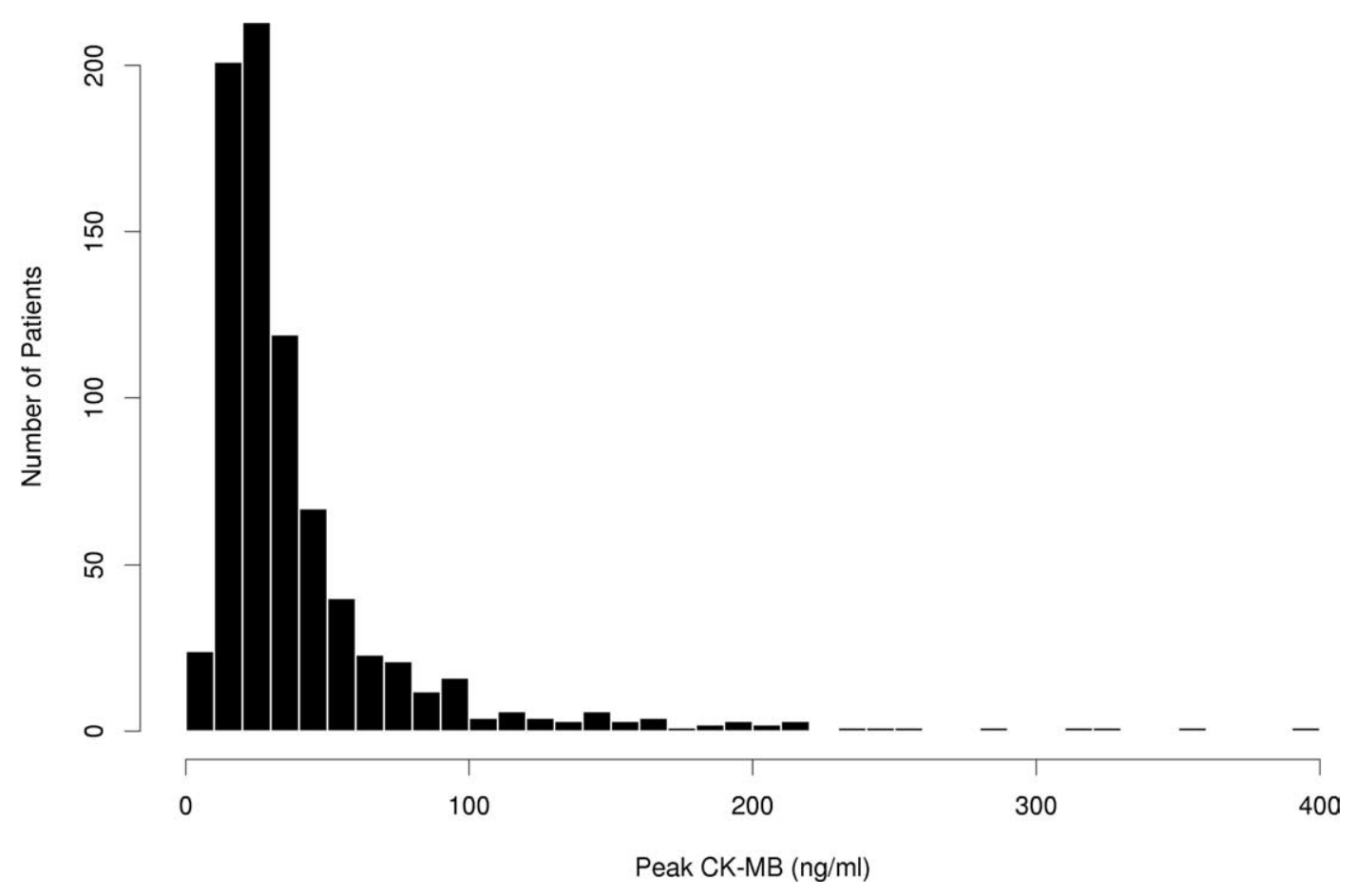

Figure 2. Distribution of peak postoperative CK-MB levels (nanograms per milliliter).

TABLE 2. Incidence of clinical adverse outcomes and 0-wave myocardial infarction according to peak postoperative CK-MB level

\begin{tabular}{|c|c|c|c|c|c|}
\hline & \multicolumn{4}{|c|}{ Peak postoperative CK-MB (ng/mL) } & \multirow[b]{2}{*}{$P$ value } \\
\hline & $0-24$ & $25-49$ & $50-99$ & $\geq 100$ & \\
\hline Death & $3 / 345(0.9)$ & $2 / 278(0.7)$ & $1 / 112(0.9)$ & $3 / 50(6.0)$ & .04 \\
\hline LVD & $3 / 343(0.9)$ & $11 / 277(4.0)$ & $5 / 112(4.5)$ & $8 / 50(16.0)$ & $<.01$ \\
\hline Death or LVD & 6/345 (1.7) & $13 / 278(4.7)$ & $6 / 112(5.4)$ & $9 / 50(18.0)$ & $<.01$ \\
\hline Q-wave $\mathrm{MI}^{*}$ & 13/335 (3.9) & $10 / 270(3.7)$ & $10 / 108(9.3)$ & $15 / 49(30.6)$ & $<.01$ \\
\hline
\end{tabular}

Data are presented as proportion of patients with outcome (\%). CK-MB, Creatine kinase MB; $L V D$, left ventricular dysfunction; $M I$, myocardial infarction. *Twenty-three patients had electrocardiogram data that were incomplete or not interpretable.

Of the 785 patients with complete CK-MB data, more than half (438/785 [55.6\%]) had peak CK-MB levels of greater than 5 times the upper limit of $5 \mathrm{ng} / \mathrm{mL}$ set by the core laboratory (Figure 2). The median peak enzyme value for the entire cohort of patients was $29 \mathrm{ng} / \mathrm{mL}$. Peak levels occurred 4 hours after release of the aortic crossclamp in 55\% of patients, 8 hours in $19 \%, 16$ hours in $12 \%$, and 20 hours in $6 \%$.

\section{CK-MB Release and Adverse Outcomes}

Trend tests revealed a significant association between postoperative peak CK-MB levels and mortality at 30 days, severe LV dysfunction, and the combination of these outcomes (Table 2). Although there is a modest increase in the incidence of these adverse outcomes for peak CK-MB levels between 25 and $49 \mathrm{ng} / \mathrm{mL}$ (5-10 times the upper limit of normal) and those between 50 and $99 \mathrm{ng} / \mathrm{mL}$ (10-20 times the upper limit of normal), the increase is markedly greater for peak CK-MB levels of $100 \mathrm{ng} / \mathrm{mL}$ or greater. Furthermore, higher peak CK-MB levels were associated with a greater likelihood of the development of new Q-wave MI on the postoperative ECG (Table 2).

\section{Other Predictors of Adverse Outcomes}

Significant predictors of 30-day mortality or severe LV dysfunction in the univariate analyses were previous CABG, CPB time, crossclamp time, Q-wave MI, and peak CK-MB level of $100 \mathrm{ng} / \mathrm{mL}$ or greater (Table 3). When multivariate analysis was performed on all univariate predictors of mortality-LV dysfunction with $P$ values of less than .15, new Q-wave MI did not contribute to the final model (Table 4). 
TABLE 3. Risk of adverse outcome (30-day mortality or early severe left ventricular dysfunction) associated with patient and perioperative factors

\begin{tabular}{lccr}
\hline Variable & Odds ratio & 95\% Cl & $\boldsymbol{P}$ value \\
\hline History of MI & 1.06 & $0.50-2.29$ & .89 \\
History of CHF & 1.80 & $0.67-4.78$ & .24 \\
History of neurologic & 1.17 & $0.48-2.88$ & .73 \\
$\quad$ event & & & \\
Previous CABG & 3.16 & $1.32-7.57$ & .01 \\
Diabetes & 1.87 & $0.95-3.68$ & .07 \\
Increased creatinine & 1.57 & $0.72-3.43$ & .26 \\
Age & 1.03 & $0.99-1.08$ & .14 \\
CPB time & 1.02 & $1.01-1.03$ & $<.01$ \\
Crossclamp time & 1.02 & $1.00-1.03$ & .01 \\
O-wave MI & 3.48 & $1.37-8.86$ & .01 \\
Peak CK-MB $\geq 100 \mathrm{ng} / \mathrm{mL}$ & 6.23 & $2.73-14.22$ & $<.01$ \\
\hline
\end{tabular}

$\mathrm{Cl}$, Confidence interval; $\mathrm{MI}$, myocardial infarction; $\mathrm{CHF}$, congestive heart failure; $C A B G$, coronary artery bypass grafting; $C P B$, cardiopulmonary bypass.

TABLE 4. Multiple logistic regression model to predict adverse outcome (30-day mortality or early severe left ventricular dysfunction)

\begin{tabular}{lccr}
\hline Variable & 0dds ratio & 95\% Cl & $\boldsymbol{P}$ value \\
\hline Previous CABG & 2.59 & $0.92-7.27$ & .07 \\
Diabetes & 2.05 & $0.98-4.29$ & .06 \\
Age & 1.05 & $1.00-1.10$ & .04 \\
CPB time & 1.02 & $1.01-1.04$ & $<.01$ \\
Peak CK-MB $\geq 100 \mathrm{ng} / \mathrm{mL}$ & 3.04 & $1.14-8.09$ & .03 \\
Q-wave MI & 2.20 & $0.71-6.79$ & .17 \\
Crossclamp time & 0.98 & $0.96-1.00$ & .10 \\
\hline
\end{tabular}

Table includes all variables with univariate $P$ values of less than 15 . $\mathrm{Cl}$, Confidence interval; $C A B G$, coronary artery bypass grafting; $C P B$, cardiopulmonary bypass; $\mathrm{MI}$, myocardial infarction.

\section{Discussion}

This analysis provides evidence that a highly increased postoperative serum CK-MB level is a strong independent predictor of severe adverse outcome after CABG. The risk of 30-day mortality or severe LV dysfunction was $18 \%$ for patients with peak CK-MB levels of $100 \mathrm{ng} / \mathrm{mL}$ or greater or 20 or more times the upper limit of normal. However, only $30.6 \%$ of these patients had new Q-wave MI, suggesting that non-Q-wave MI accounts for the majority of MIs associated with adverse outcome. Furthermore, in a multivariate analysis that included both Q-wave MI and peak CK-MB value as predictors, we found that new Q-wave MI was not an independent predictor of 30-day mortality or severe LV dysfunction. These data contradict the notion that increased postoperative CK-MB levels have less clinical utility than new Q-wave MI. Our data confirm the results of 2 other recent studies, ${ }^{8,9}$ which found that postoperative serum CK-MB levels in patients undergoing CABG are independently associated with adverse outcome.

\section{Generalization of Results}

The patient sample used in the present study consisted of 800 patients undergoing isolated CABG surgery at 65 centers throughout the United States in 1999 and 2000. This patient sample is most likely representative of the current surgical population of patients undergoing $\mathrm{CABG}$ with $\mathrm{CPB}$ for the first time. However, our results might not apply to patients who undergo off-pump CABG (OPCAB). Other randomized studies comparing $\mathrm{CABG}$ with $\mathrm{CPB}$ to $\mathrm{OPCAB}$ have demonstrated significantly less CK-MB release ${ }^{12,13}$ or troponin $\mathrm{T}$ release ${ }^{14}$ with the latter technique, but the reports provide no information regarding any relationship between these markers of myocardial ischemia and rates of mortality or severe LV dysfunction. Therefore, it is unclear whether peak CK-MB release predicts adverse outcomes in patients undergoing $\mathrm{OPCAB}$ as consistently as it does in patients who undergo $\mathrm{CABG}$ with $\mathrm{CPB}$.

\section{Prognostic Significance of CK-MB Release}

Skeletal muscles and atrial tissue both contain CK-MB. ${ }^{15,16}$ Trauma to these tissues and manipulation of the heart during CABG might induce at least some of the CK-MB release seen in these patients. Although the majority of our patients released CK-MB at levels that would indicate significant myocardial necrosis in a nonsurgical population, most did not have severe adverse cardiac outcomes, even when the peak enzyme level was up to 5 times the upper limit of normal. Similarly, in the cohort study of 99 patients undergoing on-pump CABG recently reported by Puskas and colleagues, ${ }^{13}$ the mean CK-MB level at 8 hours postoperatively was approximately $23 \mathrm{ng} / \mathrm{mL}$ ( $>4$ times the upper limit of normal), yet 30-day mortality was only $2 \%$. Therefore, these seemingly high levels of enzyme release (up to 5 times the upper limit of normal) might represent surgically associated nonischemic damage, relatively small areas of ischemia, or ischemia-reperfusion-mediated myocardial necrosis.

Long-term follow-up studies by Costa and coworkers ${ }^{8}$ and Klatte and associates ${ }^{9}$ have shown that patients with peak CK-MB release of less than 5 times the upper limit of normal continue to have a relatively low risk of mortality after the first 30 postoperative days. Costa and coworkers ${ }^{8}$ found 1 -year mortality rates of $1.1 \%, 0.5 \%, 5.4 \%$, and $10.5 \%$ for patients with peak CK-MB release of 1 or less, 1 to 3,3 to 5 , and greater than 5 times the upper limit of normal, respectively. Similarly, Klatte and associates ${ }^{9}$ found 6-month mortality rates of $3.4 \%, 5.8 \%, 7.8 \%$, and $20.2 \%$ in patients with peak enzyme levels of less than 5,5 to 10,10 to 20 , and 20 or more times the upper limit of normal, respectively. When combined with the results of the present study, these data from more than 4000 patients 
undergoing CABG between 1997 and 2000 in North America and Europe clearly demonstrate that the risk of shortand long-term mortality and other adverse outcomes increases as peak postoperative CK-MB levels increase. These data do not necessarily support the concept of a single critical level of peak enzyme release that is diagnostic for MI; rather, they suggest that the higher the peak level, the worse the prognosis. Mortality is especially high in patients with peak CK-MB levels of 20 or more times the upper limit of normal. In all 3 studies, peak CK-MB level was the strongest single predictor of adverse outcomes.

\section{CK-MB Versus Troponins}

Currently, the preferred biochemical markers for myocardial damage in the nonsurgical setting are cardiac troponins I and T., ${ }^{77}$ These enzymes are more cardiac specific than CK-MB because skeletal muscle does not contain either troponin I or $\mathrm{T}$ in significant quantities. In addition, plasma levels of the troponins remain at their peak for a longer period after myocardial damage than do CK-MB levels, making peak levels easier to determine. A few reports suggest that troponin levels are as accurate as, or even more accurate than, CK-MB levels in detecting MI (as determined by other indirect markers) in cardiac surgical patients. ${ }^{18-20}$ However, lack of power, insufficient follow-up, and other limitations prevent these studies from providing a clear estimate of the predictive value of troponins for post-CABG adverse events. In contrast, there are decades of robust data documenting the clinical specificity of CK-MB for irreversible cardiac tissue damage. ${ }^{7}$ It seems likely, however, that future studies with larger cohorts and longer follow-up will show higher troponin levels to be associated with increased risk of mortality and other adverse cardiac outcomes in patients undergoing $\mathrm{CABG}$, just as with higher CK-MB levels. Furthermore, because troponins remain at their peak levels far longer than the CK-MB isoenzyme, fewer samples might be needed to estimate risk after CABG.

\section{Relationship of ECG Findings to Peak CK-MB Level} We found a significant association between the incidence of new Q-wave MI and higher peak levels of CK-MB in that nearly one third of the patients with peak CK-MB levels of $100 \mathrm{ng} / \mathrm{mL}$ or greater had new Q-wave MI. However, although new Q-wave MI was a significant univariate predictor of 30-day mortality or severe LV dysfunction, this relationship was not sustained in the final multivariate model. Klatte and associates ${ }^{9}$ also found a significant univariate association between new Q-wave MI and 6-month mortality that fell out of the final model when multivariate analysis was performed. Thus the predictive power of peak postoperative CK-MB for post-CABG adverse outcomes predominates over that of new Q-wave MI when both factors are included in multivariate outcome analysis.
Findings from other studies also call into question the independent predictive value of new postoperative Q-wave MI. A detailed analysis and follow-up of new inferior Qwave infarctions reported in the Emory Angioplasty versus Surgery Trial ${ }^{21}$ demonstrated that 10 of 11 patients coded for such MIs had normal ejection fractions, no regional wall motion abnormalities, and patent grafts. Additionally, in 194 patients randomized to CABG surgery who were followed for 3 years, the mortality rate did not differ between patients with or without new $\mathrm{Q}$ waves $(5 \%$ vs $6.5 \%$, respectively). ${ }^{22}$ Furthermore, Klatte and associates ${ }^{9}$ found that patients who experienced peak CK-MB levels of less than 5 times the upper limit of normal had similar 6-month mortality rates irrespective of whether they had new Q-wave MI $(2.6 \%)$ or not $(3.6 \%)$. Finally, postoperative rhythm disturbances, pacing, and pericardial inflammation commonly interfere with ECG interpretation, and new Q waves have been found in some patients to reflect unmasking of old MIs. ${ }^{23}$ Although these data do not necessarily negate earlier findings that new Q-wave MI predicts adverse outcome after $\mathrm{CABG},{ }^{4,5}$ they clearly reinforce our evidence that new Q-wave MIs have little clinical significance when they coincide with low levels of CK-MB release. Alternatively, increased enzyme levels are likely to be of great importance irrespective of whether they are accompanied by new Qwave MI.

\section{Potential Limitations}

Although we believe our patient sample, together with those described by Costa and coworkers ${ }^{8}$ and Klatte and associates ${ }^{9}$ is representative of the current population of patients undergoing on-pump CABG, we acknowledge that all 3 studies involved patients enrolled in clinical trials, mostly at academic institutions. The overall population of patients with CABG might differ from this cohort in some ways; for example, they might be at lower risk for adverse outcomes. Also, the small number of adverse events might have limited the extent to which we can draw conclusions regarding the clinical significance of CK-MB increase to 5 to 10 times the upper limit of normal. Furthermore, as mentioned above, patients undergoing OPCAB were not included, and such patients generally release lower levels of CK-MB than what we observed. Additionally, our multivariate analysis included only those factors available in the database of the primary study, so that some factors that have an effect on prognosis might not have been examined. Finally, our outcome data were limited to the first 30 days after the operation; ideally, follow-up would have been longer. However, data from the 2 other large trials suggest that our primary finding, the increased risk associated with higher peak CK-MB levels, remains valid well beyond the 30-day postoperative period. 


\section{Clinical Implications}

Our findings provide robust support for the use of peak postoperative $\mathrm{CK}-\mathrm{MB}$ as an independent prognostic marker for adverse outcome in patients undergoing CABG. Furthermore, peak enzyme level is a much stronger predictor of death or LV dysfunction than is the appearance of new Q-wave MI on the ECG, especially when the new Q-wave MI is not accompanied by a substantial CK-MB increase. Because the peak might occur early or late in the postoperative period, it is probably necessary to measure this enzyme more than once during the first 24 hours after the operation. However, AUC calculation does not appear necessary. In the clinical trial setting, peak CK-MB level might be the best measure of the effectiveness of new drugs or other treatments intended to reduce the effects of CABGrelated ischemia-reperfusion injury and the inflammatory response to $\mathrm{CABG}$ and $\mathrm{CPB}$. Further study is required to determine whether troponin levels are superior to CK-MB levels in this regard. Peak CK-MB level is an effective way to identify high-risk post-CABG patients and thus permit aggressive triage and treatment to reduce the high incidence of adverse outcomes in the long term. Future studies should examine the value of specific interventions when their use is determined by postoperative CK-MB levels.

We thank Stephen N. Palmer, PhD, for his invaluable contributions to the writing of this article.

\section{References}

1. Jain U. Myocardial infarction during coronary artery bypass surgery. J Cardiothorac Vasc Anesth. 1992;6:612-23.

2. Chaitman BR, Alderman EL, Sheffield LT, et al. Use of survival analysis to determine the clinical significance of new Q waves after coronary bypass surgery. Circulation. 1983;67:302-9.

3. Force T, Hibberd P, Weeks G, et al. Perioperative myocardial infarction after coronary artery bypass surgery: clinical significance and approach to risk stratification. Circulation. 1990;82:903-12.

4. Gardner MJ, Johnstone DE, Lalonde L. Perioperative myocardial infarction with coronary artery surgery: diagnosis, incidence and consequences. Can J Cardiol. 1987;3:336-41.

5. Roberts AJ, Spies SM, Lichtenthal PR, et al. Change in left ventricular performance related to perioperative myocardial infarction in coronary artery bypass graft surgery. Ann Thorac Surg. 1983;35:516-24.

6. Califf RM, Abedelmeguid AE, Kuntz RE, et al. Myonecrosis after revascularization procedures. J Am Coll Cardiol. 1998;31:241-51.

7. Alpert JS, Antman E, Apple F, et al. Myocardial infarction redefined: a consensus document of the joint European Society of Cardiology/ American College of Cardiology committee for the redefinition of myocardial infarction. J Am Coll Cardiol. 2000;36:959-69.
8. Costa MA, Carere RG, Lichtenstein SV, et al. Incidence, predictors, and significance of abnormal cardiac enzyme rise in patients treated with bypass surgery in the arterial revascularization therapies study (ARTS). Circulation. 2001;104:2689-93.

9. Klatte K, Chaitman BR, Theroux P, et al. Increased mortality after coronary artery bypass surgery is associated with increased levels of postoperative creatine kinase-myocardial band isoenzyme release. J Am Coll Cardiol. 2001;38:1070-7.

10. Shernan SK, Fitch JCK, Nussmeier NA, et al. Impact of pexelizumab, a recombinant, humanized, single-chain, anti-C5 complement antibody, on total mortality and adverse cardiovascular outcomes in cardiac surgical patients undergoing cardiopulmonary bypass. Ann Thorac Surg. 2004;77:942-50.

11. Chaitman B, Zhou S, Tamesis B, et al. Methodology of serial ECG classification using a Minnesota code adaptation of the NOVACODE for Q-wave myocardial infarction in the Bypass Angioplasty Revascularization Investigation (BARI). J Electrocardiol. 1996;29:265-77.

12. van Dijk D, Nierich AP, Jansen EW, et al. Early outcome after off-pump vs on-pump coronary artery bypass surgery: results from a randomized study. Circulation. 2001;104:1761-6.

13. Puskas JD, Williams WH, Duke PG, et al. Off-pump coronary artery bypass grafting provides complete revascularization with reduced myocardial injury, transfusion requirements, and length of stay: a prospective randomized comparison of two hundred unselected patients undergoing off-pump versus conventional coronary artery bypass grafting. J Thorac Cardiovasc Surg. 2003;125:797-808.

14. Khan NE, De Souza A, Mister R, et al. A randomized comparison of off-pump and on-pump multivessel coronary-artery bypass surgery. N Engl J Med. 2004;350:21-8.

15. Tsung JS, Tsung SS. Creatine kinase isoenzymes in extracts of various human skeletal muscles. Clin Chem. 1986;32:1568-70.

16. Graeber GM, Cafferty PJ, Wolf RE, et al. Creatine kinase and lactate dehydrogenase in the muscles encountered during median sternotomy and in the myocardium of the cardiac chambers. J Thorac Cardiovasc Surg. 1985;89:700-5.

17. Jaffe AS, Ravkilde J, Robert R, et al. It's time for a change to a troponin standard. Circulation. 2000;102:1216-20.

18. Greenson N, Macoviak J, Krishnaswamy P, et al. Usefulness of cardiac troponin I in patients undergoing open heart surgery. Am Heart $J$. 2001;141:447-55.

19. Benoit M-O, Paris M, Silleran J, et al. Cardiac troponin I: its contribution to the diagnosis of perioperative myocardial infarction and various complications of cardiac surgery. Crit Care Med. 2001;29: 1880-6.

20. Holmvang L, Jurlander B, Rasmussen C, et al. Use of biochemical markers of infarction for diagnosing perioperative myocardial infarction and early graft occlusion after coronary artery bypass surgery. Chest. 2002;121:103-11.

21. King SB, Lembo NJ, Weintraub WS, et al. A randomized trial comparing coronary angioplasty with coronary bypass surgery. Emory Angioplasty versus Surgery Trial (EAST). N Engl J Med. 1994;331: 1044-50.

22. Hodakowski GT, Craver JM, Jones EL, King SB, Guyton RA. Clinical significance of perioperative Q wave myocardial infarction: the Emory angioplasty versus surgery trial. J Thorac Cardiovasc Surg. 1996;112: 1447-54.

23. Bassan M, Oatfield R, Hoffman I, Matloff J, Swan H. New Q waves after aortocoronary bypass surgery: unmasking of an old infarction. N Engl J Med. 1974;290:349-53. 


\section{Appendix}

\section{Pexelizumab Study Investigators}

Robert Albus, Inova Fairfax Hospital

Keith Allen, St Vincent's Medical Center

Gabriel S. Aldea, Richard Anderson, University of Washington, School of Medicine

John Armitage, Mary Washington Hospital

Clinton Baisden, Scott and White Memorial Hospital

Daniel Beckman, Richard Kovacs, Methodist Research Institute

Elliott Bennett-Guerrero, Columbia University College of Physicians \& Surgeons

Stuart Boe, North Ridge Medical Center

Michael Borkon, Mid-America Heart Institute (St Luke's)

Steven Boyce, Washington Hospital

John H. Braxton, Maine Medical Center

Clay Burnett, Memorial Medical Center, Inc

Paul Burns, Sinai Hospital

John C. Chen, Hawaii Kaiser Permanente

Richard P. Cochran, Robert Love, University of Wisconsin Hospitals and Clinics and William S. Middleton V.A. Hospital

Thomas Deal, Morton Plant Hospital

Pierre de Villiers, Cleveland Clinic Foundation

Luis Dibos, Union Memorial Hospital

Joseph Diliberto, Diagnostic Clinical Research

Francis X. Downey, St Luke's Medical Center

Mercedes Dullum, Washington Adventist

Michael P. Eaton, University of Rochester Medical Center

Fred Edwards, University of Florida Health Science Center, Jacksonville

Jack Julian Farahi, BreakThru Clinical Trials

John E. Fetter, St Mary's-Duluth Clinic

Jane Fitch, Baylor College of Medicine

Stanley A. Gall, St John's Hospital

Deepak Gangahar, Giles S. Hedderic, Nebraska Heart Institute

Michael Goldberg, Cooper Hospital UMC

Steven Goldman, Janice Christensen, Bruce Toporoff, Veteran Affairs Medical Center
L. Michael Graver, Long Island Jewish Medical Center

Michael Griffin, Yale University

Charles Hantler, University of Texas Health Sciences Center at San Antonio

Charles Hogue, Thoralf Sundt, Washington University School of Medicine

Ronald P. Karlsburg, Brotman Medical Center

Irving Kron, University of Virginia Health Science Center

Irvin Krukenkamp, Stony Brook Hospital

John H. Lemmer, Legacy Good Samaritan Hospital

Jerrold Levy, VA-Atlanta

Ted Lillehei, United Hospital

Stephen Lincoln, St Joseph's Medical Center

Stephen Longo, Pennsylvania State College of Medicine-M.S.

Hershey Medical Center

John Luber, Gilbert Johnston, St Joseph Medical

Jose Marquez, Allegheny General Hospital

Joseph P. Matthew, Andrew Hilton, Duke University Medical Center

Imran Niazi, St Francis Hospital

Nancy Nussmeier, Prashant Lotlikar, Texas Heart Institute

Dennis Pupello, Robert Goldstein, Florida West Coast Clinical Research Group

James G. Ramsay, Emory University Hospital

Jay Requarth, Camcare Health Education and Research Institute Jeffrey B. Rich, Sentara Norfolk General Hospital

Michael Rosenbloom, Memorial Regional, Hollywood, FL

Mark Sand, Definitive Health Services

Stanton K. Shernan, Brigham and Women's Hospital

Christopher Stone, Kenosha Hospital and Medical Center

John Streitz, St Luke's Hospital

Victor Tedesco, Touro Infirmary Center

James Todd, Peninsular Regional Medical Center

Melvin J. Tonkon, Anaheim Memorial

Clifford van Meter, Ochsner Foundation

Russell Vester, Lindner Center for Cardiovascular Research

Arthur Wallace, San Francisco VA Medical Center

Gary Yurow, Dale Senior, Jewish Hospital, KY

Robert Zeff, Iowa Heart Center

Kenton Zehr, Mayo Clinic 